\title{
Development of 2D and 3D Mucus Models and Their Interactions with Mucus-Penetrating Paclitaxel-Loaded Lipid Nanocapsules
}

\author{
Anne-Claire Groo Kristina Mircheva • Jérôme Bejaud • Caroline Ailhas • Ivan Panaiotov • Patrick Saulnier • Tzvetanka Ivanova • \\ Frederic Lagarce
}

Received: 16 September 2013 / Accepted: 11 December 2013 / Published online: 28 January 2014

(C) Springer Science+Business Media New York 2014

\begin{abstract}
Purpose To study, diffusion through mucus (3D model) of different formulations of paclitaxel loaded lipid nanocapsules (PtxLNCs), to interpret the results in the light of LNC behavior at air-mucus interface (2D model).

Methods LNC surface properties were modified with chitosan or poly(ethylene glycol) (PEG) coatings of different size (PEG 2,000 to 5,000 Da) and surface charges. LNC diffusion through $446 \mu \mathrm{m}$ pig intestinal mucus layer was studied using Transwell ${ }^{\circledR}$. LNCs were spread at the air-water-mucus interface then interfacial pressure and area changes were monitored and the efficiency of triglyceride (TG) inclusion was determined.

Results Ptx-LNCs of surface charges ranging from -35.7 to + $25.3 \mathrm{mV}$ were obtained with sizes between 56.2 and $75.1 \mathrm{~nm}$. The diffusion of paclitaxel in mucus was improved after encapsulation in neutral or positively charged particles $(p<0.05$ vs $\left.\operatorname{Taxo}^{(}{ }^{\circledR}\right)$. No significative difference was observed in the 2,0005,000 PEG length for diffusion both on the 2D or 3D models. On 2D model positive or neutral LNCs interacted less with mucus. Highest efficiency of TG inclusion was observed for particles with smallest PEG length.
\end{abstract}

Conclusions The results obtained with 2D and 3D model allowed us to select the best candidates for in vivo studies (neutral or positive LNCs with smaller PEG length).

KEY WORDS diffusion - mucus - nanoparticles · oral delivery . paclitaxel

A.-C. Groo · J. Bejaud • P. Saulnier • F. Lagarce

INSERM U 066 MINT «Micro et nanomédecines biomimétiques»

LUNAM Université, 49100 Angers, France

A.-C. Groo $\cdot$ C. Ailhas

Ethypharm, Chemin de la poudrière, 76121, Grand-Quevilly, France

K. Mircheva $\cdot$ I. Panaiotov $\cdot$ T. Ivanova

Biophysical Chemistry Laboratory, Department of Physical Chemistry

Faculty of Chemistry and Pharmacy, University of Sofia, J. Bourchier str. I

I 64 Sofia, Bulgaria

\section{INTRODUCTION}

Mucus covers the luminal surface of the gastrointestinal tract, respiratory, urogenital, and eyes tissues. Mucus constitutes an effective barrier against exogenous particles that can be used as drug carrier, as a consequence various studies were focused on interaction between mucus and particles. In order to improve drug diffusion through mucus, different strategies were tested, for examples mucolytic agents (1), antiacid drug coadministration (2), mucoadhesive colloidal nanosystems with chitosan (3), lectin (4) or latex particles (5), and mucuspenetrating nanoparticles. To improve mucus penetration, various surface properties modifications were used: polyelectrolyte stabilized multilayers (6), poloxamer or poly(vinyl alcohol) (7), coating, poly(ethylene glycol) (PEG) surface coverage (8), COOH-modified surface (9). Indeed, surface chemistry influences strongly nanoparticle behaviors in mucus, for example thiolated chitosan showed less bioadhesive properties than unmodified chitosan. These properties limit the occurrence of strong interactions between mucus and particle shell leaving particles still able to diffuse through the mucus (10). Mucus is particularly composed of negatively charged mucin fibers. Consequently, surface charge plays a role in the particle transport because negative charges lead to mucin-particle electrostatic repulsion and positive charges lead to attraction. Norris and Sinko (11) studied the effects of surface charge and hydrophobicity on the translocation of polystyrene

F. Lagarce

Pharmacy Department, Angers University Hospital, Angers, France

F. Lagarce $(\square)$

INSERM U 1066 MINT "Micro et nanomédecines biomimétiques "

Bâtiment IBS-IRIS, LUNAM Université, 4 rue Larrey, 49933

Angers, France

e-mail: frederic.lagarce@univ-angers.fr 
microspheres through gastric mucin. These authors found a higher permeability of amidine functionalized particles than carboxylate and sulfate functionalized particles. Crater and Carrier showed that aminemodified particle transport across the mucus was more impaired by particle-mucus interactions than carboxylate- and sulfate-modified particles (12). These different results can also be explained by other properties influencing diffusion: particle size and coating density. In the case of Crater and Carrier study, an inadequate surface coverage may increase hydrophobic interactions between hydrophobic particle cores and hydrophobic domains of the mucin. PEG-coating decreases hydrophobic interactions (9).

Over the last decade, lipid nanocapsules (LNCs) have been created and intensively used as effective drug delivery systems $(13,14)$. The LNCs are obtained by using a phase-inversion method. They contain a triglyceride (TG) core covered by a monolayer composed by phospholipids and polyethylene glycol hydroxystearate molecules (13). A post-insertion method widely applied with liposomes (15) has been also applied to modify their surface (16).

Recently the bioavailability of the Paclitaxel (Ptx) drug after encapsulation in LNCs for oral delivery has been improved by a factor 3 (17). LNCs have demonstrated an in vitro stability in artificial gastrointestinal media (18). These nanocarriers are taken up by Caco2 cells mainly via active endocytic processes. These mechanisms improved the drug gastrointestinal crossing (19) and involved a complex interaction with $\mathrm{P}$ glycoprotein (P-gp) (20). LNCs are thus a good candidate for oral administration vehicle and their stability and diffusion in intestinal mucus have recently been described (21). Indeed, Ptx diffusion improvement after encapsulation in LNGs has been demonstrated in mucus by diffusion studies across Transwell ${ }^{\circledR}$ using a 3D model, and stability was determined for $3 \mathrm{~h}$ at $37^{\circ} \mathrm{C}$ by fluorescence resonance energy transfer (FRET) (21).

In the present study, the surface of the nanocapsules was modified in order to optimize the LNG behavior in mucus. Because it is sometime difficult to correlate a particle feature to its diffusion in 3D model (as it will be discussed in this paper), a new 2D model was also implemented in order to help the selection of the best candidates for mucus diffusion. This 2D model consists of mucus monolayer obtained by spreading mucus from an aqueous solution on a surface balance. The 3D model consists of mucus layer diffusion studies: a $446 \mu \mathrm{m}$ thickness layer was deposed on Transwell ${ }^{\circledR}$ membrane.

Thus, the purpose of our paper was then to study, diffusion through the mucus (3D model) of these different formulations and to interpret the results in the light of LNC behavior at airmucus interface (2D model).

\section{MATERIALS AND METHODS}

\section{Materials}

Captex ${ }^{\circledR}$ 8,000 (tricaprylin) was obtained from Abitec Corp. (Colombus, Ohio, USA) via Unipex (Rueil-Malmaison, France). The average molecular weight was $470.7 \mathrm{~g} / \mathrm{mol}$.

Lipoid $^{\circledR}$ S75-3 (Lip) - Soybean lecithin with $69 \%$ of phosphatidylcholine (average molecular weight $800 \mathrm{~g} / \mathrm{mol}$ ) was a gift from Lipoid GmbH (Ludwigshafen, Germany).

Solutol ${ }^{\circledR}$ HS15 (Sol) - mixture of free polyethylene glycol $660(\sim 30 \%)$ and 12-hydroxystearate of polyethylene glycol $660(\sim 70 \%)$, provided by BASF (Ludwigshafen, Germany) with an average molecular weight of $911 \mathrm{~g} / \mathrm{mol}$.

Amphiphilic phospholipids PEG used in the post-insertion are supplied by Avanti ${ }^{\circledR}$ Polar Lipids Ins. (USA): 1,2 Distearoyl-sn-glycero-3 - phosphoethanolamineN-[methoxy(polyethylene-glycol)] with a PEG length 2,000 (45 units), 3,000 (68 units) and 5,000 (113 units) (DSPEPEG-OCH ${ }_{3}$,

1,2 Distearoyl-sn-glycero-3 - phosphoethanolamine-N[amino(polyethylene-glycol)] with a PEG length 2,000 (45 units) (DSPE-PEG- $\mathrm{NH}_{2}$ ); DSPE-PEG-COOH (45 units).

$\mathrm{NaCl}$ was purchased from Prolabo VWR International (Fontenay-sous-Bois, France). Paclitaxel (Ptx) powder used for LNG formulation was obtained from Teva pharm (Opava-Komarov, Czech Republic). Injectable solution of Ptx at $6 \mathrm{mg} / \mathrm{mL}\left(\right.$ Taxol $\left.^{\circledR}\right)$ was obtained from Bristol-Myers Squibb (Rueil-Malmaison, France). Purified water was obtained from a MilliQ185 System (Millipore, Paris, France). Acetonitrile, methanol, Dimethyl sulfoxide and tetrahydrofurane HPLC grade were obtained from Fisher Bioblock (Illkirch, France). Acetonitrile, methanol optima and Formic Acid optima LC-MS grade were obtained from Fisher Bioblock (Illkirch, France). PBS buffer was obtained from Lonza (Verviers, Belgium).

Sepharose ${ }^{\circledR}$ CL4-B column was obtained from SigmaAldrich (Saint-Louis, USA).

Due to the complex composition of each product, the brand names and their abbreviations will be used in the article.

\section{Preparation of Mucus}

The pigs were fasted overnight. The duodenum and jejunum of freshly slaughtered pig were isolated and cut open by ONIRIS, Nantes-France. The mucosal surfaces were washed with double-distilled water. Mucus was gently scraped with a spatula. The collected mucus were pooled and frozen in aliquots, and kept at $-20^{\circ} \mathrm{C}$ until further use. Mucus was thawed for approximately $15 \mathrm{~min}$ at room temperature before experiments. 


\section{Preparation and Characterization of Blank LNCs and Paclitaxel-Loaded LNCs}

LNGs were prepared by the phase inversion temperature method described by Heurtault et al. (2002) including several modifications. The procedure was adopted from the paper by Roger et al. (18). Briefly, a mixture of Captex ${ }^{\circledR} 8,000(29 \%$, $\mathrm{w} / \mathrm{w})$ and Lipoid ${ }^{\circledR} \mathrm{S} 75-3(1.6 \%, \mathrm{w} / \mathrm{w})$ was heated to $85^{\circ} \mathrm{C}$. Then, Solutol ${ }^{\circledR} \operatorname{HS} 15(24.15 \%, w / w), \mathrm{NaCl}(1.77 \%$, w/w) and water $(43.48 \%, \mathrm{w} / \mathrm{w})$ were added and mixed under magnetic stirring during $1 \mathrm{~min}$ at $25^{\circ} \mathrm{C}$. Then the mix was heated during $5 \mathrm{~min}$ to reach $90^{\circ} \mathrm{C}$. Three cycles of progressive heating and cooling between 70 and $90^{\circ} \mathrm{C}$ were then carried out. During the last decrease in temperature, at $78^{\circ} \mathrm{C}$, the system was diluted with $2^{\circ} \mathrm{C}$ purified water $(73 \%$, $\mathrm{v} / \mathrm{v}$ ) inducing to an irreversible shock and leading to stable LNCs. Slow magnetic stirring was then applied to the suspension of LNGs for $5 \mathrm{~min}$ at room temperature. To prepare Ptxloaded LNGs, $29.3 \mathrm{mg}$ of Ptx was solubilized into Captex ${ }^{\circledR}$ 8,000 (i.e. oil phase) and in ethanol. Then the solvent was evaporated at $85^{\circ} \mathrm{C}$. Thereafter, LNGs were then prepared as described previously. Size distributions and zeta potentials of LNCs was measured on a Zetasizer Nano series DTS 1,060 (Malvern Instruments S.A.,Worcestershire, UK). All batches were diluted 1: $60(\mathrm{v} / \mathrm{v})$ in deionized water prior to analysis, and measurements were performed three times for each experimental point at $25^{\circ} \mathrm{C}$. The size of the nanoparticles was determined by dynamic light scattering (DLS). Electrophoretic mobilities were converted to zeta potentials using Smoluchowski's equation. Precipitated non encapsulated Ptx was removed after filtration of Ptx-loaded LNCson a Minisart ${ }^{\circledR} 0.2 \mu \mathrm{m}$ filter (Vivascience AG, Hanovre, Germany). Three samples of filtrate were prepared by dissolution of an exact quantity of LNGs dispersion in a 96/4 (v/v) methanol/tetrahydrofurane solution. Ptx concentrations were then measured by high-performance liquid chromatograph (HPLC). The HPLC method was based on the method described elsewhere (18). The apparatus was composed by injector (Waters ${ }^{\circledR} 717$ plus), pump (Waters ${ }^{\circledR} 660 \mathrm{E}$ ), detector (Waters ${ }^{\circledR}$ 2,487), controller (Waters ${ }^{\circledR}$ 600), software: Millenium 32 version3.2 (Waters ${ }^{\circledR}$, Saint Quentin-enYvelines, France). The column used was an XTerra ${ }^{\circledR}$ C18ODB $150 \mathrm{~mm} \times 4.60 \mathrm{~mm}$ column (Waters, Milford, Ireland). Injected volume and run time were respectively $15 \mu \mathrm{L}$ and $45 \mathrm{~min}$. The flow rate was $1 \mathrm{~mL} / \mathrm{min}$ and the detection wavelength was $227 \mathrm{~nm}$. The gradient was obtained by mixing proportion of phase $\mathrm{A}$ (water) and phase $\mathrm{B}$ (acetonitrile). The mobile phase was initially composed of $50 \%$ B plus $50 \% \mathrm{~A}$, then reached $85 \% \mathrm{~B}$ after 7 min, maintained for $2 \mathrm{~min}$ and returned to the initial conditions, by applying a linear gradient.

The detection limit was $10.0 \mathrm{mg} / \mathrm{L}$ and the quantification limit was $20.0 \mathrm{mg} / \mathrm{L}$. The mean drug payload ( $\mathrm{mg}$ of Ptx/g of
LNG dispersion) of each batch of LNG dispersion were calculated by comparing to a calibration curve. The encapsulation efficiency $(\%)$ was calculated by consideration of the initial amount of drug added in the formulation as previously described.

\section{Preparation of Post-Inserted LNCs and Chitosan Adsorbed LNCs}

Post-inserted LNCs were prepared using the post-insertion technique (16). The blank LNG suspensions or Ptx-loaded LNCs were incubated with phospholipid-PEG surfactants for $60 \mathrm{~min}$ at $45^{\circ} \mathrm{C}$, to obtain of final concentration of $11 \mathrm{mM}$ of phospholipid-PEG. The mixture was vortexed every $15 \mathrm{~min}$, and then put in ice-bath during $1 \mathrm{~min}$. The following phospholipid-PEG surfactants were incubated with LNGs:

\section{DSPE-PEG2000-OCH \\ 2. DSPE-PEG5000- $\mathrm{OCH}_{3}$ \\ 3. DSPE-PEG2000- $\mathrm{NH}_{2}$ \\ 4. DSPE-PEG2000-COOH}

In order to eliminate free phospholipid-PEG surfactants, post-inserted blank LNCs were purified by Sepharose ${ }^{\circledR}$ CL4$\mathrm{B}$ column and concentrated on $50 \mathrm{kDa}$ Amicon ${ }^{\circledR}$ filters by centrifugation. Then the LNC concentration was calculated by absorbance at $580 \mathrm{~nm}$. The subsequent LNG interfacial modifications were confirmed by measuring sizes and zeta potentials.

To produce chitosan adsorbed LNCs, chitosan was incubated at $25^{\circ} \mathrm{C}$ during 30 min with Ptx-loaded LNCs and a small quantity of $\mathrm{NaOH} 0.1 \mathrm{~N}(1.23 \% \mathrm{v} / \mathrm{v})$ in order to limit acidification by chitosan. The final concentration of chitosan was adjusted at $5 \mathrm{mg}$ chitosan/mL of suspension.

\section{Diffusion Through the Mucus}

The transport of different formulations of Ptx: Taxol ${ }^{\circledR}$ and Ptx-loaded LNCs was studied from the donor to the receptor compartment using a Transwell ${ }^{\circledR}$ system Fig. 1a. The diffusion cell setup is made of polycarbonate Snapwell ${ }^{\circledR}$ inserts, which have an area of $1.12 \mathrm{~cm}^{2}$. PBS was placed in the receptor compartment $(1.5 \mathrm{~mL}, 10 \mathrm{mM}$ phosphate buffer, $2.7 \mathrm{mM} \mathrm{KCl} ; 137 \mathrm{mM} \mathrm{NaCl}$; pH 7.5). The test solutions were diluted in PBS at $16.8 \mu \mathrm{g} / \mathrm{mL}$ Ptx concentrations. Mucus layers were plated on the Snapwell ${ }^{\circledR}$ inserts at a volume of $50 \mu \mathrm{L}$, to obtain a mucus thickness that was calculated to be of $446 \mu \mathrm{m}$. The experiment was started by adding $0.5 \mathrm{~mL}$ of test solution at the donor side, carefully on the mucus layer. The permeability of paclitaxel "without mucus" was measured on the same systems. The donor and receptor compartments filled with PBS were separated only by the polycarbonate 
membrane. Then Transwell ${ }^{\circledR}$ was incubated at $37^{\circ} \mathrm{C}$. After $2 \mathrm{~h}$ of incubation, samples were taken from receptor compartment and Ptx content was determined by LC-MS/MS. Apparent permeability coefficient (Papp), expressed in centimeters per second, was calculated according to the following equation $(22,23)$ :

$\mathrm{P}_{\mathrm{app}}=\frac{\mathrm{dQ}}{\mathrm{AC}_{0} \mathrm{dt}}$

where $\mathrm{dQ} / \mathrm{dt}$ is the rate of drug appearance on the received side $\left(\mu \mathrm{g} . \mathrm{s}^{-1}\right), \mathrm{C}_{0}$ is the initial concentration over the donor side $\left(\mu \mathrm{g} . \mathrm{mL}^{-1}\right)$ and $\mathrm{A}$ is the surface area $\left(\mathrm{cm}^{2}\right)$. Results were expressed as mean values \pm SD. For statistical analysis, a Student's t-test was used and $p<0.05$ was considered statistically significant.

\section{Ptx Analysis by LC-MS/MS}

The procedure was adopted from the paper by Roger et al. (19). Chromatography was performed using a Waters Alliance ${ }^{\circledR} 2,695$ system (Waters S. A.) with an Uptisphere ${ }^{\circledR}$ C18ODB $150 \times 2.0 \mathrm{~mm}, 5 \mu \mathrm{m}$ column (Interchrom, Montluçon, France). The gradient profile of mobile phase of $0.1 \%$ formic acid in water / $0.1 \%$ formic acid in methanol used was generated according to a gradient timetable. The flow rate was $0.3 \mathrm{~mL} / \mathrm{min}$ and the column temperature was set at $40.0^{\circ} \mathrm{C}$. The total HPLC effluent was directed into a Quattro Micro ${ }^{\circledR}$ triple quadruple mass spectrometer (Waters S. A.). Multiple reaction monitoring $(\mathrm{MRM})$ mode and turbo ion spray in positive ion mode wer used. The $(\mathrm{M}-\mathrm{H})+\mathrm{m} / \mathrm{z}$ transitions for Ptx were $854.1 \rightarrow 286.1$ and $876.3 \rightarrow 308.1$ (sodium adduit), and the typical retention time was $6.41 \mathrm{~min}$. Ptx concentrationwas calculated with QuantLynx ${ }^{\circledR}$ (Waters S.A.) by comparison to a calibration curve. The range of linear response was $0.015-7.2 \mu \mathrm{g} / \mathrm{mL}$. The lower limit of detection and the lower limit of quantification were $0.015 \mu \mathrm{g} / \mathrm{mL}$ and $0.75 \mu \mathrm{g} / \mathrm{mL}$, respectively.

\section{Measurement at Air-Water Interface}

The surface pressure $(\boldsymbol{\pi})$ was measured using KSV-2200 (Finland) surface balance, equipped with platinum plate and a Teflon ${ }^{\circledR}$ trough with an area of $475 \mathrm{~cm}^{2}$. In the case of preformed films from mucus, the monolayers were obtained by spreading mucus from an aqueous solution. A period of about $30 \mathrm{~min}$ was necessary for formation of a stable surface film. Once the film surface was stable, the LNC dispersion was spread (Fig. 1b). All measurements were performed at room temperature.

In order to characterize the state of the mucus layer, the equilibrium isotherms $\pi(\mathrm{A})$ of the mucus monolayer is measured. After spreading and formation of a stable surface film at low surface pressure $\left(\pi<0.2 \mathrm{mN} . \mathrm{m}^{-1}\right)$ the films were compressed by means of a movable barrier with a sufficiently low velocity $\left(\mathrm{U}_{\mathrm{b}}=10.8 \mathrm{~cm}^{2} \cdot \mathrm{min}^{-1}\right)$ to stay in a linear regime.

In order to follow the interfacial disaggregation of nanocapsules, two types of experiments were performed with LNGs or modified LNGs spreading:

- $\quad$ Recording the surface pressure $(\pi)$ change with time $(\mathrm{t})$ at constant surface area (A);

- $\quad$ Recording of the surface area change $(\Delta \mathrm{A})$ with time $(\mathrm{t})$ at constant surface pressure $(\pi)$;

The disaggregation of nanocapsules might lead to a rapid release of TG from the core of LNCs and to the formation of mixed TC/mucus monolayers.

\section{Interpretation of the Experimental Data $\pi\left(t_{\mathbf{A}}=\right.$ const and $\triangle A(t)_{\pi=\text { const }}$}

The interpretation of the experimental data $\Delta \mathrm{A}(\mathrm{t})_{\pi=\text { const }}$ and $\pi(t)_{\mathrm{A}=\text { const }}$ data (Fig. 5) can be obtained on the basis of the following model. A rapid disaggregation of the unstable LNCs leads to release of TG molecule, on the previously formed mucus monolayer. The TG molecules were previously confined in LNC capsules. During the formation of a mixed TG/ mucus monolayer, the total mass of both species spread at the whole available surface area is given by the following simple expression:

$m(t)=m_{1}(t)+m_{2}(t)=\Gamma_{1}(t) \frac{A(t) M_{T G}}{\mathcal{N}_{A}}+m_{2}(t)$

where $m_{1}(\mathrm{t})$ and $m_{2}(\mathrm{t})$ are mass of TG and mucus, respectively.

Whole available area mixed monolayer; $A(t)=A_{0}+\Delta A(t)$; $\Gamma_{1}(t)$ is the surface concentration of TG (number of molecules per unit area); $\mathrm{M}_{\mathrm{TG}}$ is the molecular mass; $\mathrm{N}_{\mathrm{A}}$ is the Avogadro number

$\underline{\text { At constant surface area }\left(\mathrm{A}_{0}=\text { const }\right)} m_{2}^{0}=$ const and Eq. (1) is reduced to

$m(t)=\frac{A_{0} M_{T G}}{\mathcal{N}_{A}} \Gamma_{1}(t)+m_{2}^{0}$

The rate of the total mass increase is proportional to the rate of increase of the TG molecules surface concentration in the mixed TG/mucus monolayer

$\frac{d m}{d t}=\frac{A_{0} M_{T G}}{\mathcal{N}_{A}} \frac{d \Gamma_{1}}{d t}$ 
a

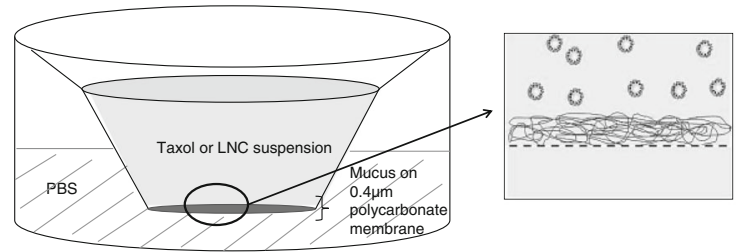

b

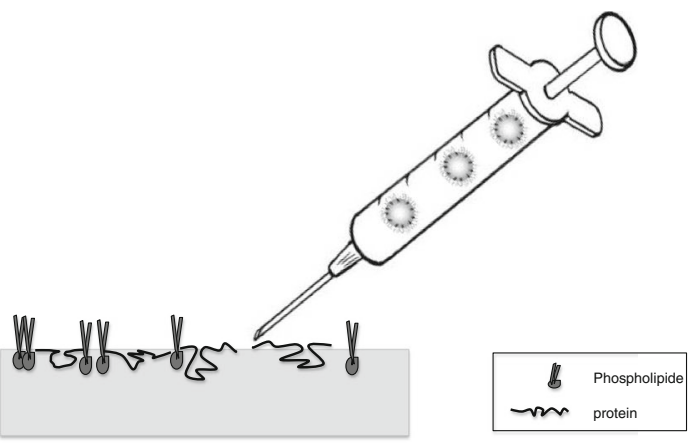

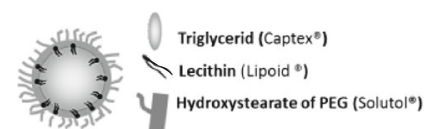

Fig. I 3-D (a) and 2-D (b) mucus membrane models.

We assume that the process of disaggregation of the unstable capsules is rate dependent, and that the spreading of the TG molecules confined into the capsules is instantaneous. The following kinetic equation for the initial rate of increase of the surface concentration of TG molecules in the mixed monolayer can be written as:

$$
\left(\frac{d \Gamma_{1}}{d t}\right)_{i n i}=k_{1}(1-\theta) m_{1}^{0} \mathcal{N}
$$

where $k_{1}$ is the rate constant of LNC disaggregation.

$\Gamma_{2}^{\infty}$ corresponds to the observed maximal packing in the mucus monolayer. At the beginning of the process, $\Gamma_{1}(t)=0 . \quad \theta=\frac{\Gamma_{2}}{\Gamma_{2}^{\infty}} \quad$ is the degree of surface packing in the preformed mucus monolayer; $(1-\theta)$ is the part of free surface area; $\mathcal{N}$ is the number of spread LNCs per unit area; $m_{1}^{0}$ is the number of TG molecules confined in one LNG; $m_{1}^{0} \mathcal{N}$ is the number of TG molecules contained in all LNCs spread at the unit surface.

As a consequence

$$
\left(\frac{d \Gamma_{1}}{d t}\right)_{i m i}=\frac{\Gamma_{2}^{0}}{E_{2}^{0}}\left(\frac{d \pi}{d t}\right)_{i m i}
$$

where $E_{2}^{0}=\frac{d \pi}{d \Gamma_{2}} \Gamma_{2}^{0}$ is the surface elasticity of the previously spread mucus monolayer. From Eqs. (4) and (5) one obtains the following expression for the rate constant $k_{1}$ :

$$
k_{1}=\frac{(d \pi / d t)_{i n i} \Gamma_{2}^{0}}{E_{2}^{0}(1-\theta) m_{1}^{0} \mathcal{N}}
$$

\section{Case of a Constant Surface Pressure ( $\pi=$ Const $)$}

Assuming the ideal behavior of the formed mixed TG/mucus monolayer, we can consider that the initial surface area $A_{0}$ is only covered by mucus. The observed surface area change $(\Delta \mathrm{A})$ is only attributed to TG molecules with constant surface density $I_{1}^{0}$. Then, the Eq. (1) is reduced to:

$m(t)=\Delta A(t)\left(M_{T G} / \mathcal{N}_{A}\right) \Gamma_{1}^{0}+m_{2}^{0}$

From Eq. (7) we can determine the values for the maximal degree of inclusion $d_{\mathrm{TG}}$ of TG in the mucus monolayer as the ratio between the mass of both species TG and mucus in the mixed monolayer

$d_{T G}=\frac{m_{1}^{\text {sat }}}{m_{2}^{0}}=\frac{\Delta A_{\text {sat }}\left(M_{T G} / \mathcal{N}_{A}\right) \Gamma_{1}^{0}}{m_{2}^{0}}$

The corresponding inclusion efficiency $\varepsilon_{\mathrm{TG}}$ is the ratio between the mass of TG included in the surface film and those contained in all spread LNCs

$\varepsilon_{T G}=\frac{m_{1}^{\text {sat }}}{m_{1}^{0} \mathcal{N} A}=\frac{\Delta A_{\text {sat }}\left(M_{T G} / \mathcal{N}_{A}\right) \Gamma_{1}^{0}}{m_{1}^{0} \mathcal{N} A}$

$\varepsilon_{\mathrm{TG}}=1$ when all $\mathrm{TG}$ molecules contained in all spread LNCs are included in the surface monolayer.

The inclusion degree depends strongly on the surface pressure of the previously spread monolayer and could be as usually characterized also by the maximal insertion pressure (MIP). The plot of $\Delta \pi$ as a function of $\pi_{\mathrm{i}}$ (see Fig. 6).allows the determination of the MIP by extrapolating the regression of the plot to the $\mathrm{x}$ axis. 


\section{RESULTS AND DISCUSSION}

\section{Preparation and Characterization of Blank LNCs, Post-Inserted Blank LNCs and Ptx-Loaded LNCs}

The resulting physical characteristics of the LNCs are presented in Table I. The size increases with post-insertion and chitosan adsorption. Zeta potentials were negative for LNCs coated with DSPE-PEG-OCH 3 and LNCs coated with DSPE-PEG-COOH. It was almost zero for LNGs coated with DSPE-PEG-NH $\mathrm{N}_{2}$ as well as standard LNCs. It was positive for LNCs covered with chitosan. For all formulations the polydispersity index $(\mathrm{PDI})$ was $<0.2$ which demonstrates the monodispersity of the preparations.

Encapsulation efficiency of Ptx in various LNGs (Table I) was higher than $95 \%$ and the drug payload ranged from 1.83 to $1.98 \mathrm{mg} / \mathrm{g}$ of formulation.

\section{Diffusion Phenomena from Different Formulations Through the Mucus Thick Layer}

Diffusion possibilities of Ptx-loaded LNCs are presented on Fig. 2. Three hypotheses could be proposed for this diffusion process: 1 - diffusion of intact Ptx-loaded LNCs, 2 - diffusion of Ptx associated to remnants of LNCs, 3 - diffusion of Ptx alone released after LNC disaggregation. The first hypothesis is the most likely for two reasons. First, LNG stability was determined for $3 \mathrm{~h}$ at $37^{\circ} \mathrm{C}$ by fluorescence resonance energy transfer, FRET (21). Secondly, Ptx is extremely lipophilic $(\log \mathrm{P}=3.5)$ and thus is not able to easily diffuse in mucus and in mucus lipids component.
The permeability through the mucus of Ptx encapsulated in LNGs, with different LNG surface properties versus commercial Ptx $\left(\right.$ Taxol $\left.^{\circledR}\right)$, is presented in Fig. 3. LNCs improved Ptx diffusion by a factor 2 compared to Taxol ${ }^{\circledR}$. Taxol ${ }^{\circledR}$ micelles showed 8-20 nm spherical structures (24). This small size did not explain that Taxol ${ }^{\circledR}$ diffusion was impeded by mucus. As a consequence, diffusion was hindered by interaction between micelles and mucus. Yudin et al revealed that mucus has a fibrous structure with a $500 \mathrm{~nm}$ interfiber spacing between the primary elements and an additional finer structure with a spacing of around $100 \mathrm{~nm}$ (25). Because studied LNCs had a size of $55 \mathrm{~nm}$, steric hindrance may not stop LNC diffusion. However, diffusion depended on interaction between LNCs and mucus. As a consequence, Ptx permeability was found dependent on the LNG surface properties.

Positive or neutral LNGs (LNGs, LNGs with chitosan and LNCs coated with DSPE-PEG2000amino) could go through the mucus better than the most negative LNCs (LNGs coated with DSPE-PEG2000carboxy). In agreement with this result, Norris and Sinko (11) found that the more positive is the zeta potential, the higher is the diffusion. Dawson and al. demonstrated that cationic nanoparticles have a higher transport rates (26). Lieleg, Vladescu and Ribbeck also showed the importance of the surface potential on diffusion (27). In fact, charged particles can interact by electrostatic interaction with the mucin, which contained numerous acidic and basic amino acids. Diffusion is allowed for neutral particle. On the contrary, when nanoparticles exhibited a hydrophobic surface, which interacted with the hydrophobic domains of the mucin chains, negative charge could be beneficial. Mura et al observed that positive or neutral PLGA nanoparticles were entrapped by mucus whereas negative PLGA nanoparticles

Table I LNC Properties (A, Blank LNCs and B, Ptx-loaded LNCs)

\begin{tabular}{|c|c|c|c|c|c|}
\hline & & Size, Z-average (nm) & PDI & Zeta potential (mV) & LNC concentration $(\mathrm{mg} / \mathrm{mL})$ \\
\hline \multicolumn{2}{|l|}{ Blank LNCs } & $54.6 \pm 0.6$ & $0.024 \pm 0.009$ & $-5.4 \pm 0.3$ & 153 \\
\hline \multicolumn{2}{|l|}{ LNC-DSPE-PEG2000-amino } & $64.7 \pm 0.4$ & $0.057 \pm 0.012$ & $3.7 \pm 0.3$ & 48 \\
\hline \multicolumn{2}{|l|}{ LNC-DSPE-PEG2000-carboxy } & $68.0 \pm 0.4$ & $0.071 \pm 0.024$ & $-41.4 \pm 0.6$ & 48 \\
\hline \multicolumn{2}{|l|}{ LNC-DSPE-PEG2000-methoxy } & $61.8 \pm 0.4$ & $0.062 \pm 0.017$ & $-27.6 \pm 0.2$ & 81 \\
\hline \multicolumn{2}{|l|}{ LNC-DSPE-PEG5000-methoxy } & $70.9 \pm 0.4$ & $0.060 \pm 0.008$ & $-17.8 \pm 0.3$ & 30 \\
\hline \multicolumn{6}{|l|}{ B. } \\
\hline & Size, Z-average $(\mathrm{nm})$ & PDI & Zeta potential (mV) & Drug payload (mgPtx/g) & Loading efficiency (\%) ${ }^{a}$ \\
\hline Ptx-LNCs & $56.2 \pm 1.0$ & $0.038 \pm 0.005$ & $-7.6 \pm 1.3$ & 1.98 & 99.8 \\
\hline LNC-DSPE-PEG2000-amino & $61.7 \pm 0.9$ & $0.064 \pm 0.021$ & $4.3 \pm 0.9$ & 1.89 & 95.7 \\
\hline LNC-DSPE-PEG2000-carboxy & $64.1 \pm 0.6$ & $0.081 \pm 0.006$ & $-35.7 \pm 3.3$ & 1.89 & 95.3 \\
\hline LNC-DSPE-PEG2000-methoxy & $62.6 \pm 0.9$ & $0.077 \pm 0.008$ & $-27.2 \pm 1.6$ & 1.88 & 95.1 \\
\hline LNC-DSPE-PEG3000-methoxy & $65.5 \pm 0.6$ & $0.070 \pm 0.003$ & $-20.4 \pm 2.7$ & 1.87 & 94.4 \\
\hline LNC-DSPE-PEG5000-methoxy & $75.1 \pm 0.8$ & $0.102 \pm 0.016$ & $-16.5 \pm 0.7$ & 1.83 & 92.4 \\
\hline LNCs with chitosan & $67.1 \pm 0.4$ & $0.182 \pm 0.004$ & $25.3 \pm 1.9$ & 1.89 & 95.3 \\
\hline
\end{tabular}

${ }^{a}$ Theoretical drug loading was $1.98 \mathrm{mgPtx} / \mathrm{g}$ 
Fig. 2 Alternative ways to describe the experimentally observed diffusion of Ptx through the mucus layer.

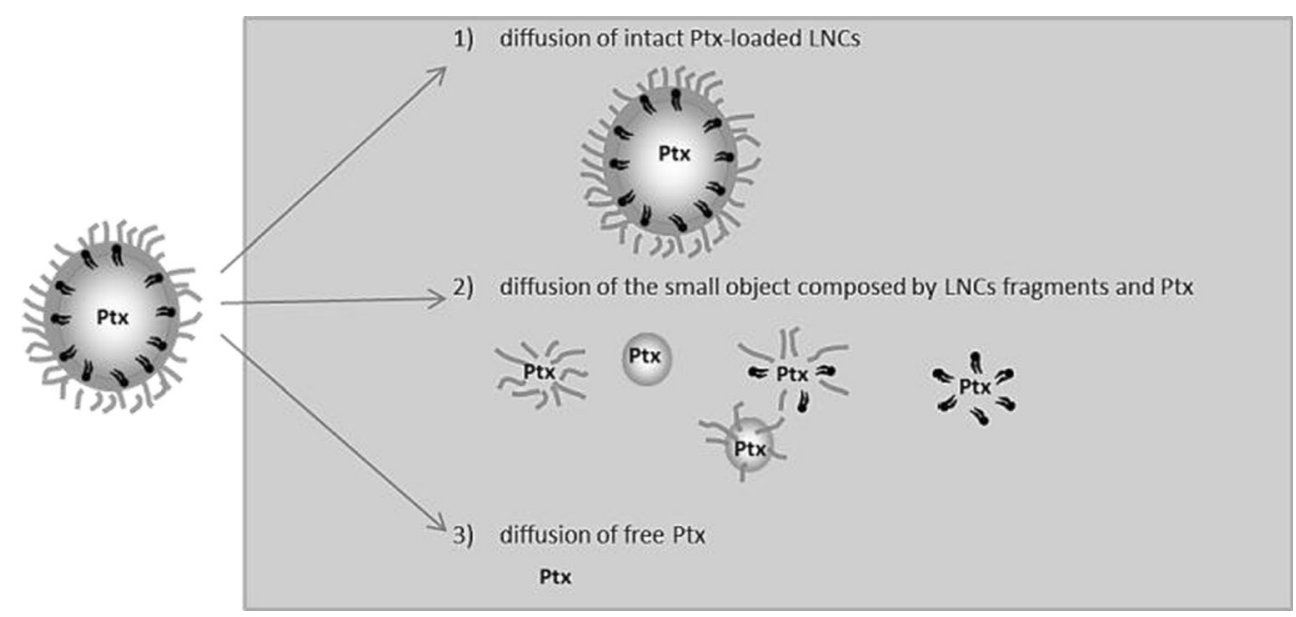

diffused freely in the mucin networks. Hydrophobic interactions were balanced by electrostatic repulsions (7). LNC shell was composed of PEG and lecithin phospholipids, so their external surface presented hydrophilic characteristics where no hydrophobic interaction was expected. As a consequence, no electrostatic repulsion was needed for diffusion and was indeed not desirable. Thus carboxy and methoxy nanocapsules were not considered as optimal. On the contrary, neutral charge particles avoided electrostatic interaction and improved diffusion. No significative differences was found between neutral and positive particles in our study. Thus, we think that for a good diffusion of hydrophilic small

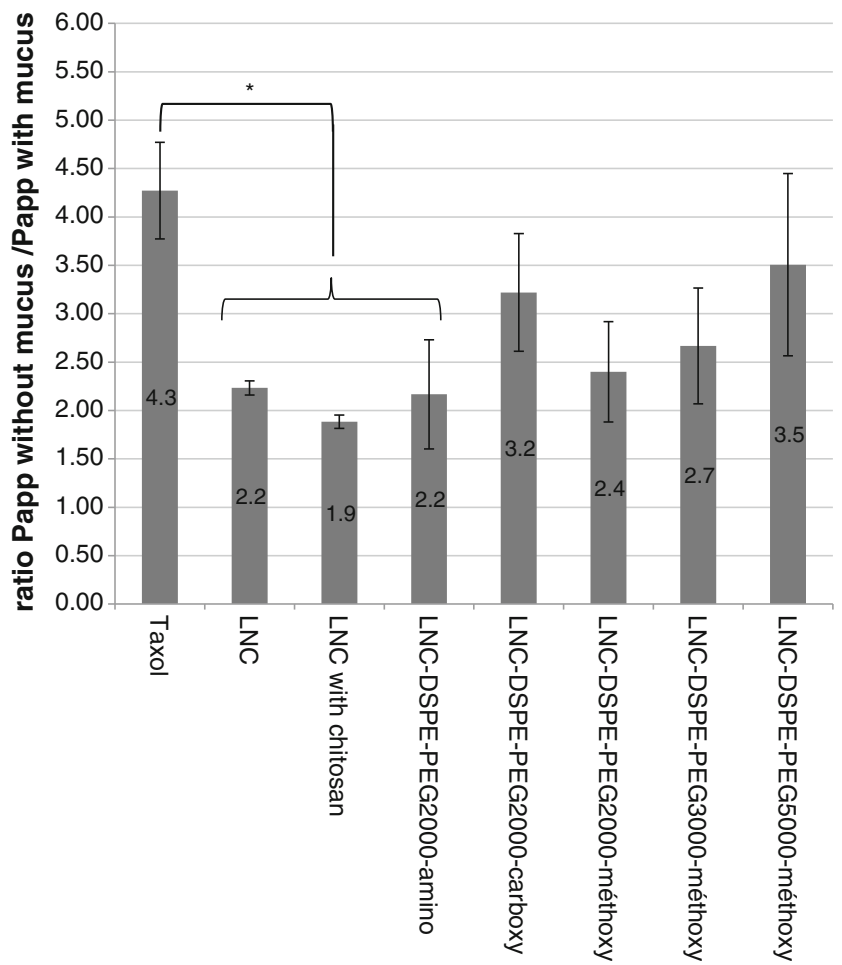

Fig. 3 Effect of LNC surface modification on diffusion through the mucus $(n=3) . * p<0.05$. nanocarriers, neutral or positively charged surfaces are to be sought for good mucus diffusion properties.

In order to avoid both hydrophobic and electrostatic interactions, mucus penetrating particles (MPP) were coated with PEG, a hydrophilic and uncharged polymer. This coating minimized efficiently particle adhesion to mucus constituents (28). Coating particles with PEG may reduce these particlemucus adhesive interactions, if the molecular weight (MW) of PEG was sufficiently low to avoid adhesion by polymer interpenetration and hydrogen bonding (29-31). PEG with low MW adopted a brush conformation that could facilitate their diffusion in the mucus by hindering the hydrophobic interactions (30,31). On the opposite, the disposition of the longer PEG chains (i.e. $10 \mathrm{kDa}$ ) was different at the nanoparticle surface and would favor the interpenetration and interaction with the mucus fibers (29). PEGs with too low MW, for example $1 \mathrm{kDa}$, were distributed inside or physically adsorbed on the nanoparticle surface.

A dense covalent coating with low MW PEG led to particles penetrating more easily in human cervico-vaginal mucus (9). In our work, standard LNC formulations were coated by PEG660 (Solutol HS15). LNGs were also coated with longer chains of PEG in order to assess the role of PEG length on the ability of LNCs to diffuse across mucus. In order to find the optimal MW of PEG coating on LNCis, 3 PEG methoxy MW were tested: $2 \mathrm{kDa}, 3 \mathrm{kDa}$ and $5 \mathrm{kDa}$.

The mucus slowed down the LNCs coated with DSPEPEG5000methoxy diffusion and this diffusion was not found significantly different from Taxol ${ }^{\circledR}$ diffusion unlike LNGs coated with DSPE-PEG2000-methoxy. So the chain length seems also to slightly influence diffusion. However, no significant difference was observed but a trend may be brought out: the longer the PEG chain was, the slower the diffusion was across mucus. As a consequence, PEG $2 \mathrm{kDa}$ seems to be the most favorable coating.

In our work crude intestinal mucus from pork was used. In fact, purified mucin solution cannot provide an accurate 
model of native mucus because it does not contain all mucus constituents, which increased the possible interactions between particles and environment. For example, Larhed et al studied diffusion of different drugs through native pig intestinal mucus (PIM) and purified pig gastric mucin (PPGM) (32). LNCs were intended to be used for human oral delivery, and so needed to overcome intestinal mucus. Pig mucus and human mucus are similar in structure and molecular weight (33). As a consequence, pig intestinal mucus model was used in order to evaluate LNG 3D diffusion. In addition to differences in mucus composition; differences in mucus structure were also described in the literature and can play a role in particle and drug diffusion. For all particles, particle transport rates were much more heterogeneous in native mucus, due to the higher heterogeneity of the mucus mesh porosity (12). At small scale level, this heterogeneity was negligible. For example, when microscopic motion of hundreds of particles were studied during some seconds or over a distance of a few micrometers by using multiple particle tracking (9), a low variabitity was observed. But, this heterogeneity may influence particle diffusion when time and distance increase. Then, many research teams observed an experimental variability by using modified side-by-side diffusion cell with a thickness of mucus layer above hundreds $\mu \mathrm{m}$ (11). A mucus monolayer model (2D) may smooth this heterogeneity and decrease variability in order to focus on interaction between mucus and particles.

Diffusion decreased due to the interaction between mucus and particles. The variability of particle permeability was higher for smaller diffusion particles. Trends emerged from Fig. 3 but conclusion cannot be draught

a

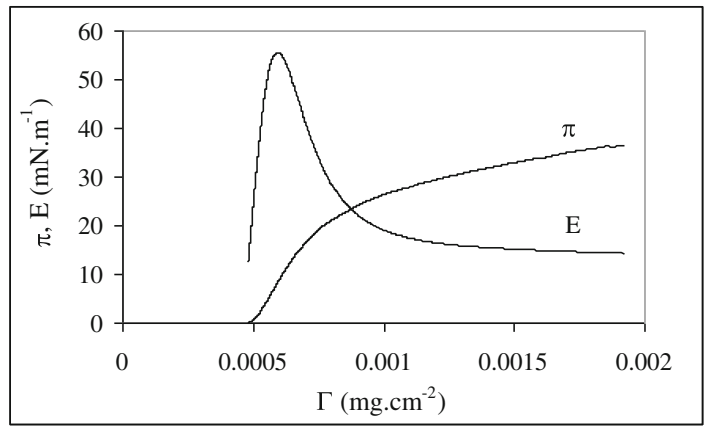

b

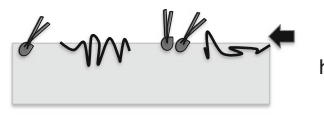

heterogenic mixed layer $(<7 \mathrm{mN} / \mathrm{m})$

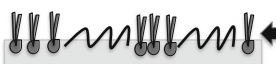

inflexion point $(\sim 7 \mathrm{mN} / \mathrm{m})$
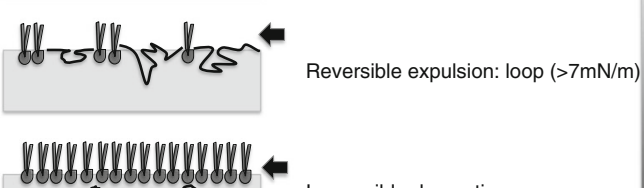

है दे Irreversible desorption

1 Compression

7 protein

Fig. 4 Surface pressure $(\pi)$-surface density $(Г)$ and surface elasticity $(E)$-surface density $(\ulcorner)$ of mucus monolayer (a); Scheme of a monolayer composed by lipid and proteins (b). 
coated with DSPE-PEG5000-OCH ${ }_{3}$ and LNCs coated with DSPE-PEG2000-NH $\mathrm{NH}_{2}$ LN on the whole monolayer area covered with preformed mucus surface film (0.17 mg mucus) are presented in Fig. 5. The values for $\mathrm{d}_{\mathrm{TG}}, \varepsilon_{\mathrm{TG}}$ and $\mathrm{k}_{1}$, calculated with the equations described above, are presented in Table II.
Fig. 5 Interaction of LNCs at airwater-mucus interface at a constant area $\mathrm{A}$ (left column of graphs) and at a constant pressure $\pi$ (right column of graphs). $\pi=5$ (curves I), $\pi=10$ (curves 2), $\pi=15$ (curves 3) and $\pi=20$ (curves 4) of LNCs (a and b); LNCs coated with DSPEPEG2000-OCH 3 (c and d); LNCs coated with DSPE-PEG5000$\mathrm{OCH}_{3}$ (e and $\left.\mathbf{f}\right)$ and LNCs coated with DSPE-PEG2000- $\mathrm{NH}_{2}$ (gand h) and LNCs coated with -DSPEPEG2000-COOH (I and $\mathbf{m})$.

\section{a}

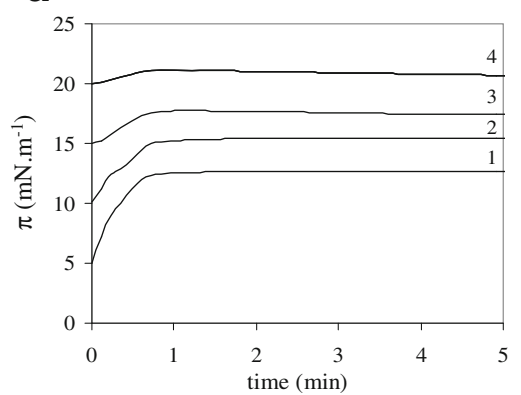

C

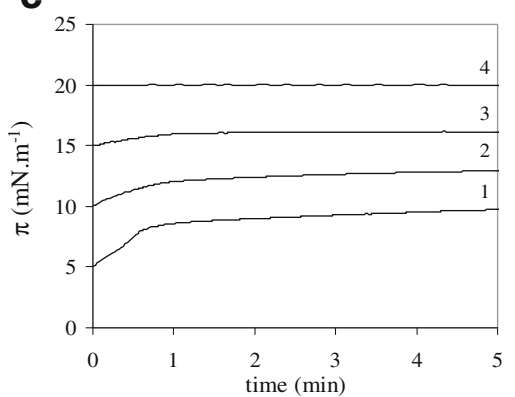

e

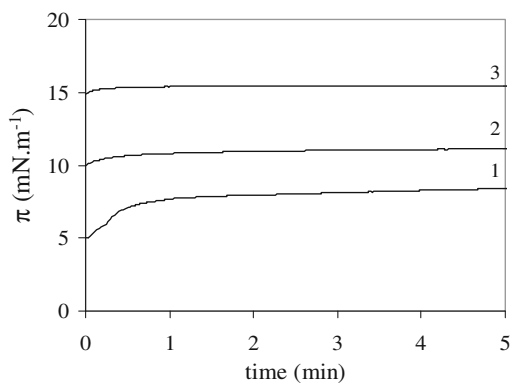

g

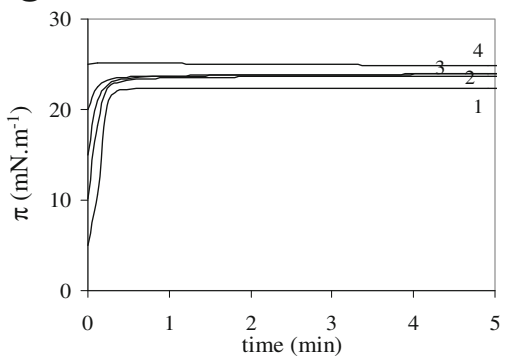

I

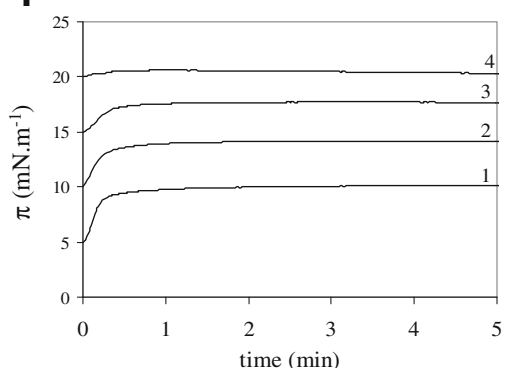

b

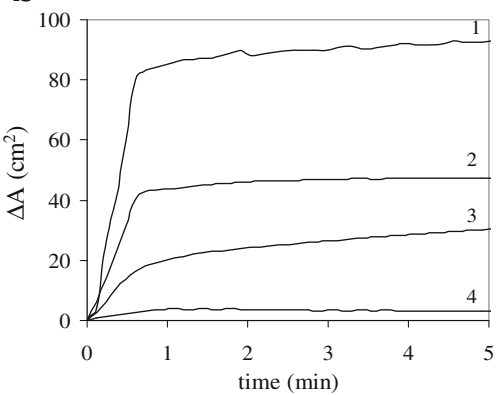

d

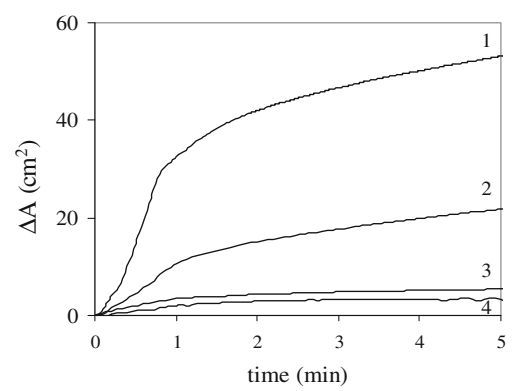

f

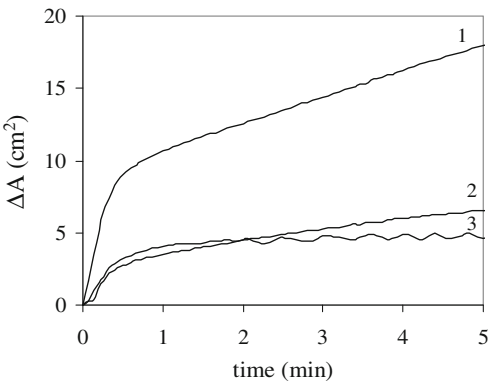

h

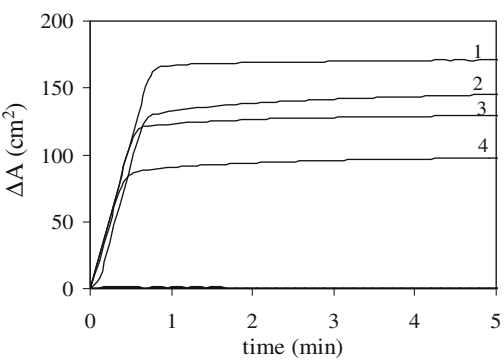

m

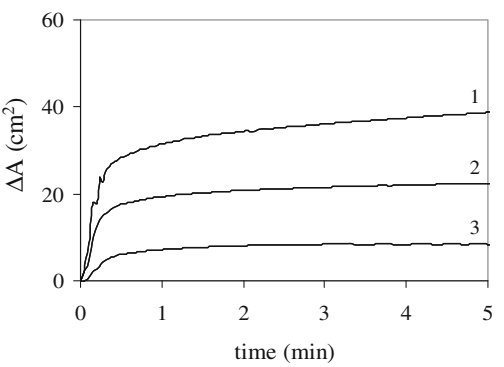


The efficiency $\varepsilon_{\mathrm{TG}}$ of TG inclusion as a result of nanocapsule disaggregation brings additional information about the LNCs - mucus monolayer interactions. LNGs interact with mucus and when they are placed at the air-mucus interface, the high surface pressure leads to a partial LNC disaggregation. As LNCs are stable in mucus at least $3 \mathrm{~h}$ at $37^{\circ} \mathrm{C}$ we can conclude that the observed LNC disaggregation is only due to the higher interfacial pressure that prevails at the air-mucus interface.

Concerning post-inserted LNCs, $\varepsilon_{\mathrm{TG}}$ decreases for LNCs coated with DSPE-PEG2000-OCH ${ }_{3}$ and especially for LNCs coated with DSPE-PEG5000-OCH 3 , while increases for LNCs coated with DSPE-PEG2000- $\mathrm{NH}_{2}$. A high $\varepsilon_{\mathrm{TG}}$ is a sign of the LNC ability to penetrate into the mucus monolayer.

The particle shell is charged negatively for LNCs coated with DSPE-PEG-OCH${ }_{3}$ and positively for $\mathrm{LNC}$ s coated with DSPE-PEG- $\mathrm{NH}_{2}$, while the mucus monolayer is mainly composed of negatively charged molecules. Positive or neutral LNCs interacted less with mucus, so exhibited the higher efficiency of TG inclusion. On the contrary, electrostatic repulsion occurring between negatives LNCs and mucus, explained lower efficiency of TG inclusion. LNCs coated with DSPE-PEG2000-carboxy were more negative than LNCs coated with DSPE-PEG2000-methoxy (OCH3) with the same PEG chain, and have also the smaller inclusion efficiency at $5 \mathrm{mN} \cdot \mathrm{m}^{-1}$. Higher negative charge led to a higher repulsion interaction. But this difference was very small; PEG chain length has a higher impact on inclusion efficiency. A lower interaction between mucus layer and nanoparticles pegylated with lower MW PEG chains explained the findings. That is in accordance with diffusion results (3D). Similarly, Zabaleta et al. found a higher apparent permeability of particles pegylated with PEG 2 or PEG $6 \mathrm{kDa}$ than nanoparticles pegylated with PEG $10 \mathrm{kDa}$ (34). The 2D system could be a new simple complementary tool to $3 \mathrm{D}$ diffusion.

However, zeta potentials of LNGs coated with DSPEPEG2000-OCH 3 and LNGs coated with DSPEPEG5000- $\mathrm{OCH}_{3}$ were different $(-27.6 \mathrm{mV}$ and $-17.8 \mathrm{mV}$ respectively) which can influence interaction. As seen before, the more negative the zeta potential was, the stronger repulsion interactions were. Even if a stronger repulsive interaction due to zeta potential was expected for LNCs coated with DSPE-PEG2000-OCH ${ }_{3}$, TG of LNGs coated with DSPE-PEG2000- $\mathrm{OCH}_{3}$ were more incorporated in mucus layer. That confirmed that the higher inclusion efficiency for LNGs coated with DSPE-PEG2000- $\mathrm{OCH}_{3}$ was also due to a smaller molecular PEG chain.

Ptx LNCs were used for diffusion assays whereas blank LNCs were spread in 2D model. However, similar properties were obtained for both types of LNCs. Ptx was lipophilic and was solubilized into LNC lipophilic core. Ptx-loaded LNCs surface was identical to blank LNCs surface. As a consequence, the same behavior in mucus was expected for both types of LNCs.

The degree of inclusion depends strongly of the surface pressure of the previously spread monolayer and is usually characterized by so-called maximal insertion pressure (MIP).

Table II Interactions of Small LNCs Quantities on Preformed Mucus Monolayer (2D Model)

\begin{tabular}{|c|c|c|c|}
\hline $\begin{array}{l}\text { Surface pres- } \\
\text { sure } \pi_{\text {mucus }}^{0} \\
{\left[\mathrm{mN} \cdot \mathrm{m}^{-1}\right]}\end{array}$ & $\begin{array}{l}\text { Maximal degree of inclu- } \\
\text { sion } \text { TTG of TG in the mu- }_{\text {cus monolayer }}\end{array}$ & $\begin{array}{l}\text { Efficiency } \\
\varepsilon_{\mathrm{TG}} \text { of TG } \\
\text { inclusion }^{\mathrm{a}}\end{array}$ & $\begin{array}{l}\text { Disaggregation } \\
\text { rate constant } k_{\mathrm{I}} \\
{\left[\mathrm{min}^{-1}\right]}\end{array}$ \\
\hline \multicolumn{4}{|l|}{ A. } \\
\hline 5 & 0.032 & 0.49 & 7.4 \\
\hline 10 & 0.018 & 0.29 & 5.5 \\
\hline 15 & 0.009 & 0.14 & 3.3 \\
\hline 20 & 0.002 & 0.03 & 3.2 \\
\hline 25 & 0.000 & 0.00 & 0.0 \\
\hline \multicolumn{4}{|l|}{ B. } \\
\hline 5 & 0.013 & 0.21 & 2.6 \\
\hline 10 & 0.006 & 0.10 & 1.5 \\
\hline 15 & 0.002 & 0.03 & 0.9 \\
\hline 20 & 0.002 & 0.03 & 0.0 \\
\hline 25 & 0.000 & 0.00 & 0.0 \\
\hline \multicolumn{4}{|l|}{ C. } \\
\hline 5 & 0.005 & 0.07 & 2.0 \\
\hline 10 & 0.002 & 0.03 & 1.1 \\
\hline 15 & 0.001 & 0.01 & 0.9 \\
\hline 20 & 0.000 & 0.00 & 0.0 \\
\hline 25 & 0.000 & 0.00 & 0.0 \\
\hline \multicolumn{4}{|l|}{ D. } \\
\hline 5 & 0.059 & 0.92 & 45.6 \\
\hline 10 & 0.055 & 0.86 & 46.5 \\
\hline 15 & 0.058 & 0.90 & 47.7 \\
\hline 20 & 0.051 & 0.79 & 43.7 \\
\hline 25 & 0.001 & 0.01 & 0.00 \\
\hline \multicolumn{4}{|l|}{ E. } \\
\hline 5 & 0.011 & 0.17 & 10.9 \\
\hline 10 & 0.008 & 0.12 & 8.2 \\
\hline 15 & 0.003 & 0.05 & 5.7 \\
\hline 20 & 0.002 & 0.03 & 3.9 \\
\hline 25 & 0.000 & 0.00 & 0.0 \\
\hline
\end{tabular}

Spread on the whole surface area quantities: TG - 0.011 mg; mucus 0.17 mg; A: LNC; B: LNCs coated with DSPE-PEG2000-OCH3; C: LNCs coated with DSPE-PEG5000-OCH3; D: LNCs coated with DSPEPEG2000-NH2; E: LNCs coated with DSPE-PEG2000-COOH

${ }^{a}$ See text for calculus details 
Fig. 6 Penetration of TG from disaggregated LNCs (a) from disaggregated LNCs coated with DSPE-PEG2000- $\mathrm{NH}_{2}$ (b); LNCs coated with DSPE-PEG2000-

$\mathrm{OCH}_{3}(\mathbf{c})$ and LNCs coated with DSPE-PEG5000-OCH 3 (d) into mucus monolayer. MIP the maxima insertion pressure. a

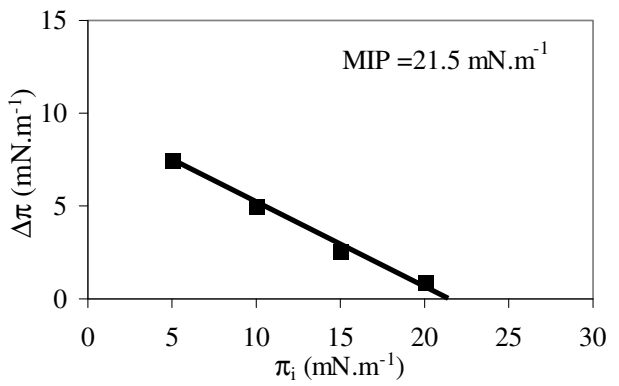

C

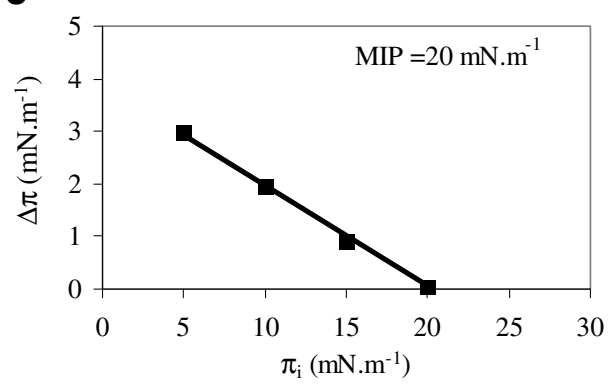

b

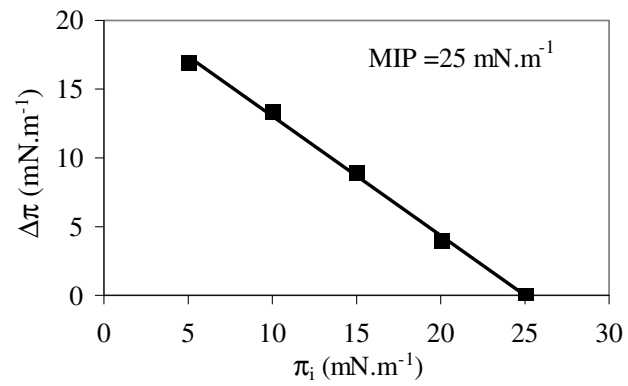

d

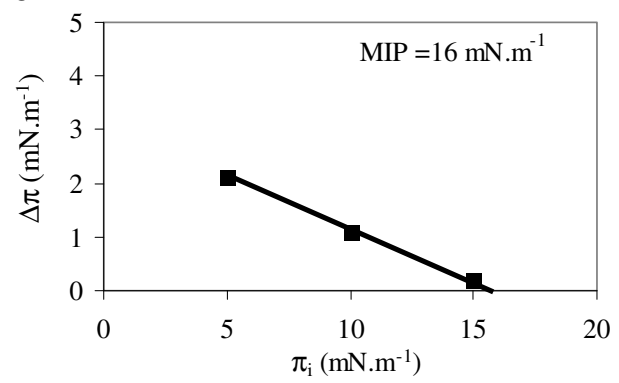

Some examples for the data $\Delta \pi\left(\pi_{\mathrm{i}}\right)$ and the obtained values for MIP are presented in Fig. 6. The MIP for the penetration of $\mathrm{TG}$ into mucus monolayer is larger for $\mathrm{LNC}_{\mathrm{s}}$ coated with DSPE-PEG-NH $\mathrm{N}_{2}$ than for the LNCs coated with DSPE-PEG-OCH$H_{3}$. This result is in agreement with the results for the efficiency $\varepsilon_{\mathrm{TG}}$ of nanocapsule penetration.

Mucin is known to have surfactant property (35). The properties of adsorbed mucin at the solid-liquid interface were first studied by Proust et al. in the mid-1980s (36). Mucus contains mainly, water (around 95\%), glycoproteins (i.e. mucin) and lipids (0.5-5\%), mineral salts $(0.51 \%)$ and free proteins $(1 \%)(37)$. Based on all these observations, crude mucus was spread in order to obtain more adequate model than mucin. An important advantage of the monolayer approach is the ability to control and modify the interfacial organization of the spread molecule by variation of the surface pressure and density.

Previous studies showed that the LNCs were stable and remain intact in the bulk of the aqueous suspensions but undergo a rapid disaggregation at air-water interface leading to the formation of a surface film (38). The formed surface film contained mainly oil and Solutol, a part of LNC constituents (39). Kinetic was different for PEG and oil adsorption. PEG and distearoyl-sn-glycero-3-phosphoethanolamine (DSPE)PEG were hydrophilic and so solubilized in sub phase. At the first minutes, only oil formed monolayer at the the air/water interface. LNC disaggregation was proportional to oil spread at the interface and PEG or
DSPE-PEG did not influence interfacial pressure. As a consequence, disaggregation of the LNCs and postinserted LNGs with various DSPE-PEG can be compared at the interface air-water-mucus. However, chitosan have a surface activity at the interface airphospholipid (40). In addition, chitosan interacted with mucin at the air liquid interface (41). Consequently, the disaggregation of LNGs with chitosan cannot be compared with those of LNGs and post-inserted LNGs. The comparison between the results for the permeability of Ptx -loaded LNGs with different modified surfaces (Fig. 2) and efficiency $\varepsilon_{\mathrm{TG}}$ of nanocapsules disaggregation (Table II) seems to argue for a diffusion improvement through the mucus model by surface properties modification. The developed air-mucus interfacial model even that is not occurring in the intestinal barrier, allows the selection of the more suitable particles for $3 \mathrm{D}$ mucus layer diffusion. Higher is the disaggregation rate in $2 \mathrm{D}$ greater is the apparent permeability in $3 \mathrm{D}$.

\section{CONCLUSION}

LNG 2D behavior at the air-mucus interface and LNG 3D behavior in mucus were studied for different type of LNCs and showed their complementarity. LNC behavior at airwater-mucus interface gave information about interfacial interactions between the mucus and LNGs; whereas 3D model allows seeing results of interaction between mucus and LNCs on diffusion phenomenon. Diffusion through the mucus $(3 \mathrm{D}$ 
model) of particles is a complex phenomenon depending on mucus model, size and surface properties of particles.

The 2D model allowed to better interpreting diffusion results. Interfacial method constituted an efficient screening method and allowed us to select the most favorable LNC formulations for mucus penetration and diffusion. In fact the formulations that best crossed mucus layer were positive or neutral and did not exhibit longer PEG chains. Consequently, the selected LNCs for the next in viwo studies were standard LNCs, LNCs coated with DSPE-DSPE-PEG2000-amino and LNCs coated with chitosan. The next step of this work will be to conduct an in vivo experiment in order to study the effect of particle surface over bioavailability. In viwo/ in vitro correlation could thus be envisioned.

\section{REFERENCES}

1. Takatsuka S, Kitazawa T, Morita T, Horikiri Y, Yoshino H. Enhancement of intestinal absorption of poorly absorbed hydrophilic compounds by simultaneous use of mucolytic agent and non-ionic surfactant. Eur J Pharm Biopharm. 2006;62(1):52-8.

2. Shaw LR, Irwin WJ, Grattan TJ, Conway BR. The influence of excipients on the diffusion of ibuprofen and paracetamol in gastric mucus. Int J Pharm. 2005;290(1-2):145-54.

3. Mistry A, Glud SZ, Kjems J, Randel J, Howard KA, Stolnik S, et al. Effect of physicochemical properties on intranasal nanoparticle transit into murine olfactory epithelium. J Drug Target. 2009;17(7):54352.

4. Ezpeleta I, Arangoa MA, Irache JM, Stainmesse S, Chabenat G, Popineau Y, et al. Preparation of Ulex europaeus lectin-gliadin nanoparticle conjugates and their interaction with gastrointestinal mucus. Int J Pharm. 1999;191(1):25-32.

5. Szentkuti L. Light microscopical observations on luminally administered dyes, dextrans, nanospheres and microspheres in the preepithelial mucus gel layer of the rat distal colon. J Control Release. 1997;46(3):233-42.

6. Jain S, Kumar D, Swarnakar NK, Thanki K. Polyelectrolyte stabilized multilayered liposomes for oral delivery of paclitaxel. Biomaterials. 2012;33(28):6758-68.

7. Mura S, Hillaireau H, Nicolas J, Kerdine-Romer S, Le Droumaguet B, Delomenie C, et al. Biodegradable nanoparticles meet the bronchial airway barrier: how surface properties affect their interaction with mucus and epithelial cells. Biomacromolecules. 2011;12(11): 4136-43.

8. Lai SK, Wang YY, Hanes J. Mucus-penetrating nanoparticles for drug and gene delivery to mucosal tissues. Adv Drug Deliv Rev. 2009;61(2):158-71.

9. Lai SK, O’Hanlon DE, Harrold S, Man ST, Wang YY, Cone R, et al. Rapid transport of large polymeric nanoparticles in fresh undiluted human mucus. Proc Natl Acad Sci U S A. 2007;104(5):1482-7.

10. Bravo-Osuna I, Vauthier C, Chacun H, Ponchel G. Specific permeability modulation of intestinal paracellular pathway by chitosanpoly(isobutylcyanoacrylate) core-shell nanoparticles. Eur J Pharm Biopharm. 2008;69(2):436-44.

11. Norris DA, Sinko PJ. Effect of size, surface charge, and hydrophobicity on the translocation of polystyrene microspheres through gastrointestinal mucin. J Appl Polym Sci. 1997;63(11):1481-92.
12. Grater JS, Carrier RL. Barrier Properties of Gastrointestinal Mucus to Nanoparticle Transport. Macromol Biosci. 2010;10(12):1473-83.

13. Heurtault B, Saulnier P, Pech B, Proust JE, Benoit JP. A novel phase inversion-based process for the preparation of lipid nanocarriers. Pharm Res. 2002;19(6):875-80.

14. Anton N, Gayet P, Benoit JP, Saulnier P. Nano-emulsions and nanocapsules by the PIT method: an investigation on the role of the temperature cycling on the emulsion phase inversion. Int J Pharm. 2007;344(1-2):44-52.

15. Allen TM, Sapra P, Moase E. Use of the post-insertion method for the formation of ligand-coupled liposomes. Cell Mol Biol Lett. 2002;7(2):217-9.

16. Perrier T, Saulnier P, Fouchet F, Lautram N, Benoit JP. Postinsertion into Lipid NanoCapsules (LNCs): From experimental aspects to mechanisms. Int J Pharm. 2010;396(1-2):204-9.

17. Peltier S, Oger JM, Lagarce F, Couet W, Benoit JP. Enhanced oral paclitaxel bioavailability after administration of paclitaxel-loaded lipid nanocapsules. Pharm Res. 2006;23(6):1243-50.

18. Roger E, Lagarce F, Benoit JP. The gastrointestinal stability of lipid nanocapsules. Int J Pharm. 2009;379(2):260-5.

19. Roger E, Lagarce F, Garcion E, Benoit JP. Lipid nanocarriers improve paclitaxel transport throughout human intestinal epithelial cells by using vesicle-mediated transcytosis. J Control Release. 2009;140(2):174-81.

20. Roger E, Lagarce F, Garcion E, Benoit JP. Reciprocal competition between lipid nanocapsules and P-gp for paclitaxel transport across Caco-2 cells. Eur J Pharm Sci. 2010;40(5):422-9.

21. Groo A-C, Saulnier P, Gimel J-C, Gravier J, Ailhas C, Benoit J-P, et al. Fate of Paclitaxel lipid nanocapsules in intestinal mucus in view of their oral delivery. Int J Nanomedecine. 2013;8(1):4291-302.

22. Artursson P, Borchardt RT. Intestinal drug absorption and metabolism in cell cultures: Caco-2 and beyond. Pharm Res. 1997;14(12): 1655-8.

23. Artursson P, Karlsson J. Correlation between oral drug absorption in humans and apparent drug permeability coefficients in human intestinal epithelial (Caco-2) cells. Biochem Biophys Res Commun. 1991;175(3):880-5.

24. SzebeniJ. Complement activation-related pseudoallergy: A new class of drug-induced acute immune toxicity. Toxicology. 2005;216(2â $€^{\prime \prime 3): 106-21 . ~}$

25. Yudin AI, Hanson FW, Katz DF. Human cervical mucus and its interaction with sperm: a fine-structural view. Biol Reprod. 1989;40(3):661-71.

26. Dawson M, Krauland E, Wirtz D, Hanes J. Transport of polymeric nanoparticle gene carriers in gastric mucus. Biotechnol Prog. 2004;20(3):851-7.

27. Lieleg O, Vladescu I, Ribbeck K. Characterization of particle translocation through mucin hydrogels. Biophys J. 2010;98(9): 1782-9.

28. Lai SK, Suk JS, Pace A, Wang YY, Yang M, Mert O, et al. Drug carrier nanoparticles that penetrate human chronic rhinosinusitis mucus. Biomaterials. 2011;32(26):6285-90.

29. Huang Y, Leobandung W, Foss A, Peppas NA. Molecular aspects of muco- and bioadhesion: Tethered structures and site-specific surfaces. J Control Release. 2000;65(1-2):63-71.

30. Yoncheva K, Guembe L, Campanero MA, Irache JM. Evaluation of bioadhesive potential and intestinal transport of pegylated poly(anhydride) nanoparticles. Int J Pharm. 2007;334(1-2):156-65.

31. Yoncheva K, Lizarraga E, Irache JM. Pegylated nanoparticles based on poly(methyl vinyl ether-co-maleic anhydride): preparation and evaluation of their bioadhesive properties. Eur J Pharm Sci. 2005;24(5):411-9.

32. Larhed AW, Artursson P, Gråsjö J, Björk E. Diffusion of drugs in native and purified gastrointestinal mucus. J Pharm Sci. 1997;86(6): $660-5$. 
33. Kararli TT. Comparison of the gastrointestinal anatomy, physiology, and biochemistry of humans and commonly used laboratory animals. Biopharm Drug Dispos. 1995;16(5):351-80.

34. Zabaleta V, Ponchel G, Salman H, Agueros M, Vauthier C, Irache JM. Oral administration of paclitaxel with pegylated poly(anhydride) nanoparticles: Permeability and pharmacokinetic study. EurJ Pharm Biopharm. 2012;81(3):514-23.

35. Shi L, Miller C, Caldwell KD, Valint P. Effects of mucin addition on the stability of oil-water emulsions. Colloids Surf B: Biointerfaces. 1999;15(3-4):303-12.

36. Proust JE, Baszkin A, Boissonnade MM. Adsorption of bovine submaxillary mucin on surface-oxidized polyethylene films. J Colloid Interface Sci. 1983;94(2):421-9.

37. Khanvilkar K, Donovan MD, Flanagan DR. Drug transfer through mucus. Adv Drug Deliv Rev. 2001;48(2-3):173-93.
38. Minkov I, Ivanova T, Panaiotov I, Proust J, Saulnier P. Reorganization of lipid nanocapsules at air-water interface: I. Kinetics of surface film formation. Colloids Surf B: Biointerfaces. 2005;45(1):14-23.

39. Minkov I, Ivanova T, Panaiotov I, Proust J, Saulnier P. Reorganization of lipid nanocapsules at air-water interface: Part 2. Properties of the formed surface film. Colloids Surf B: Biointerfaces. 2005;44(4):197-203.

40. Pavinatto FJ, Caseli L, Pavinatto A, Dos Santos DS, Nobre TM, Zaniquelli MED, et al. Probing Chitosan and Phospholipid Interactions Using Langmuir and Langmuir-Blodgett Films as Cell Membrane Models. Langmuir. 2007;23(14):7666-71.

41. Silva CA, Nobre TM, Pavinatto FJ, Oliveira Jr ON. Interaction of chitosan and mucin in a biomembrane model environment. J Colloid Interface Sci. 2012;376(1):289-95. 